\title{
Postoperative Radiotherapy in Head and Neck Cancer
}

\author{
Vedang Murthy, Sayan Kundu, Tanweer Shahid, Ashwini Budrukkar, Tejpal Gupta, \\ Sarbani Ghosh Laskar, Jaiprakash Agarwal
}

Department of Radiation Oncology, Advanced Center for Treatment Research and Education in Cancer (ACTREC) and Tata Memorial Hospital, Dr Ernest Borges Marg, Parel, Mumbai 400012, Maharashtra, India

Correspondence: Vedang Murthy, Assistant Professor, Department of Radiation Oncology, Advanced Center for Treatment Research and Education in Cancer (ACTREC) and Tata Memorial Hospital, Dr Ernest Borges Marg, Parel, Mumbai 400012, Maharashtra, India, Phone: 022 27405000, e-mail: vmurthy@actrec.gov.in

\begin{abstract}
Though early stage head and neck cancers can be cured either by surgery or radiation, patients with locally advanced disease continues to pose a therapeutic challenge. Locoregional failure is the major cause of death in head and neck cancers. As the outcome of locally advanced head and neck cancer is less than promising, a combined modality approach is generally undertaken in this group of patients. The combination of surgery, radiation and more recently, chemotherapy and targeted therapy can improve outcomes in locally advanced head and neck cancer patients. This overview discusses the rationale and role of postoperative radiotherapy (PORT) in advanced head and neck cancers, the radiotherapy technique in brief and methods of enhancing the efficacy of postoperative RT by altering the fractionation schedules and adding chemotherapy and targeted therapy.

Keywords: Head and neck cancer, postoperative, adjuvant, radiotherapy.
\end{abstract}

\section{INTRODUCTION AND RATIONALE}

Head and neck cancers are common in India and account for about $30 \%$ of cancers in males and about $13 \%$ in females. In males, oral cavity and pharynx are the commonly affected sites, followed by larynx. In females, oral cavity is the preponderant site. ${ }^{1}$ Nearly $60 \%$ of the patients present with locally advanced disease. Locoregional failure constitutes the predominant recurrence pattern, and most deaths result from uncontrolled local and/or regional disease.

Surgery and radiation, used alone or in combination, are the only curative treatments. Approximately, 80-90\% of patients with stage I or II head and neck cancer are cured with surgery or radiotherapy (RT) alone. Outcomes for patients with locally advanced stage III to IVB head and neck cancer are less promising ${ }^{2}$ and they almost always need a combination of surgery, radiotherapy and more recently chemotherapy and targeted therapy.

Locoregional control (LRC) is critical in the management of head and neck malignancies as salvage of locoregional disease is often difficult. The multimodality approach in locally advanced SCCHN has been found to reduce the risk of local failure and improve survival. ${ }^{3,4}$ Though RT alone or with concurrent chemoradiation has proven to be successful for organ preservation in patients with low volume disease, those with locally advanced cancers have a low chance of cure even with aggressive chemoradiation. Surgery is the preferred initial treatment for majority of these patients and adjuvant radiation is recommended to enhance the likelihood of LRC.

Inspite of improvement in therapeutic approaches, the outcome with multimodality combinations of surgery, radiotherapy, and chemotherapy, the 5-year overall survival rate for patients with advanced disease is approximately $50 \% .^{5}$ This is mainly due to suboptimal LRC and a low probability of successful salvage surgery. ${ }^{6}$ The rationale for postoperative RT is to prevent locoregional recurrence (recurrence at the primary site and/or neck) by destroying the microscopic deposits of cancer cells in the vicinity of operative bed.

This review discusses the rationale and role of postoperative radiotherapy (PORT) in advanced head and neck cancers, the radiotherapy technique in brief and methods of enhancing the efficacy of postoperative RT by altering the fractionation schedules and adding chemotherapy and targeted therapy.

\section{INDICATIONS: WHEN SHOULD PORT BE GIVEN?}

The use of radiotherapy in addition to surgery in locally advanced head and neck cancer began in the middle of 20th 
century. Since then, multiple retrospective trials have documented improvement in local control, disease free survival and overall survival for the patients receiving adjuvant radiotherapy. ${ }^{7}$ A phase III trial conducted by Radiation Therapy Oncology Group (RTOG 73-03) randomized 354 advanced head and neck cancer patients to preoperative or postoperative radiotherapy to determine the most effective regimen. Results from this study showed a statistically significant improvement in LRC (48\% vs 63\%; $\mathrm{p}=0.03)$ and improved survival $(26 \%$ vs $38 \% ; \mathrm{p}=0.04)$ for the postoperative arm in oropharyngeal cancers. This trial established PORT as the sequencing of choice in head and neck cancers. ${ }^{8}$

The appropriate selection of patients for PORT has also been the subject of much research. In general, PORT is given in those patients who have a more than $15-20 \%$ chance of failure in the neck or primary site. Peters et al from MD Anderson cancer center identified pathological features that put patients at high-risk of failure. ${ }^{9}$ Ang et al reported a prospective trial of 213 patients with locally advanced oral cavity, oropharynx, larynx and hypopharynx cancers treated with surgery and randomized by risk factors to postoperative radiation. Risk factors included $>1$ nodal group, $\geq 2$ nodes, nodes $>3 \mathrm{~cm}$, microscopic positive margins, perineural invasion, oral cavity site and extracapsular extension. Low risk patients were those with no risk factors and were not given postoperative radiation. Intermediate risk patients had only 1 risk factor (but not ECE) and were given 57.6 Gy. High-risk patients had ECE or $\geq 2$ risk factors and were given $63 \mathrm{~Gy}$. The 5 year LRC/OS for low-risk patients were $90 \%$ or $83 \%$, that of intermediate risk patients was $94 \%$ or $66 \%$ and for high-risk was $68 \%$ or $42 \% .^{10}$

The high-risk features include microscopically positive surgical margins, extracapsular extension, lymphovascular invasion, perineural invasion, $\geq 2$ involved neck nodes, $>1$ positive nodal group, nodal diameter $>3 \mathrm{~cm},>6$ week interval between surgery and radiation and oral cavity primary site. Although there continues to be variability between institutions, these risk factors are used as criteria for giving postoperative radiation. Other important factors are advanced T stage, recurrent disease, tumor spillage, multicentricity, and invasion of bone, cartilage, skin or soft tissue of the neck. Based on an analysis of local recurrences in RTOG trials, RTOG currently defines high-risk as the presence of positive surgical margins, extracapsular extension, or multiple lymph nodes. ${ }^{11}$ Depth of tumor invasion has been reported to be associated with a higher-risk of lymphatic spread. Tumor depth over $3 \mathrm{~mm},{ }^{12} 4 \mathrm{~mm}^{13,14}$ and $5 \mathrm{~mm}^{15}$ have been advocated as an indication of PORT. Patients with one or more of the above risk factors merit PORT.

\section{EFFICACY: DOES POSTOPERATIVE RT IMPROVE LOCOREGIONAL CONTROL AND SURVIVAL?}

Postoperative radiation in high-risk head and neck cancer patients is usually recommended to enhance the likelihood of LRC. Evidence for its usefulness comes from a small randomized trial a few other studies that have addressed the issue in different ways. Mishra et al conducted a prospective randomized trial of surgery with or without adjuvant radiation 6 weeks after surgery in oral cancers. They reported a 30\% absolute improvement in disease-free survival, although there was no difference in overall survival with the use of adjuvant radiation therapy. ${ }^{16}$ Other wellconducted (but nonrandomized studies) also point towards a benefit with adjuvant PORT.

A study conducted by Medical College of Virginia deserves special mention. ${ }^{3}$ Head and neck cancer patients were operated by two groups of surgeons. The general surgeons did not send the operated patients for radiation and reserved radiation only for recurrence. The ENT surgeons always sent high-risk head and neck cancer patients for radiation. A total of 441 patients were treated surgically between 1982 and 1988 out of which 125 had extracapsular extension and/or positive margins. Of them, 71 were treated with surgery alone and 54 received postoperative radiation. At 3 years follow-up, the local control rates for surgery alone compared to postoperative radiation were $31 \%$ and $66 \%(P=0.03)$ for extracapsular extension, $41 \%$ and $49 \%$ $(P=0.04)$ for positive margins and $0 \%$ and $68 \%(P=$ $0.001)$ for extracapsular extension and positive margins respectively.

Lundahl et al, in another series administered postoperative radiation to 95 patients of node positive squamous cell cancers of head and neck. A matched-pair analysis was performed with another series that treated patients with surgery alone. The rates of recurrence in the dissected neck $(\mathrm{RR}=5.82 ; P=0.0002)$, recurrence in either side of the neck $(\mathrm{RR}=2.21 ; P=0.0052)$, and death from any cause ( $\mathrm{RR}=1.67 ; P=0.0182)$ were significantly higher for patients treated with surgery alone. ${ }^{4}$

Mishra et al from, Cuttack, India in a prospective randomized trial have shown the superiority of adding 
postoperative radiation in locally advanced buccal mucosa cancers. ${ }^{16} 140$ patients of stage III and IV buccal mucosa cancers were randomized to surgery alone $(n=60)$ and surgery followed by postoperative radiation $(n=80)$. After a follow-up of 3 years, the disease free survival was found to be $38 \%$ and $68 \%(\mathrm{p}<0.005)$ respectively. In another series, Frank et al showed statistically significant LRC by PORT over surgery alone in 110 patients with locally advanced hypopharyngeal cancers. ${ }^{17}$

Although, PORT improves outcomes in high-risk patients, results still remain suboptimal. The PORT trials have shown a local failure rate of $30 \%, 25 \%$ rate of distant metastases and overall survival of $30-40 \%$ at 5 years. None of the trials have shown an improvement in overall survival with PORT in locally advanced head and neck cancers. But as recurrence at local and regional site remains the most common form of treatment failure and dismal rates of successful salvage treatment even in the best of hands, postoperative radiation should be strongly recommended in locally advanced head and neck cancer with high-risk features.

\section{TREATMENT TECHNIQUES: HOW IS IT CARRIED OUT BY THE RADIATION ONCOLOGIST?}

\section{Preparation of Patient for Treatment}

All patients have dental evaluation and extraction of carious or loose teeth at least 7-10 days prior to the start of treatment. This is done as any traumatic manipulation of the bone upto 1-2 years post RT can precipitate osteoradionecrosis. Nutritional assessment by a dietician prior to commencement of RT and insertion of a feeding tube may help if oral intake in inadequate due to the surgical deformity. Patients are advised to abstain from smoking and drinking alcohol and counseled regarding the expected toxicity.

\section{Immobilization}

To ensure daily reproducibility of treatment set up, patients have to be positioned such that the area to be treated is immobilized. For the head and neck region a thermoplastic mask is commonly used that has the ability to mould itself to the patient's anatomic contour and provide fixation of the area (Fig. 1). A rubber traction helps in retracting the shoulders out of the radiation beam and provides stability to the arms (Fig. 2).

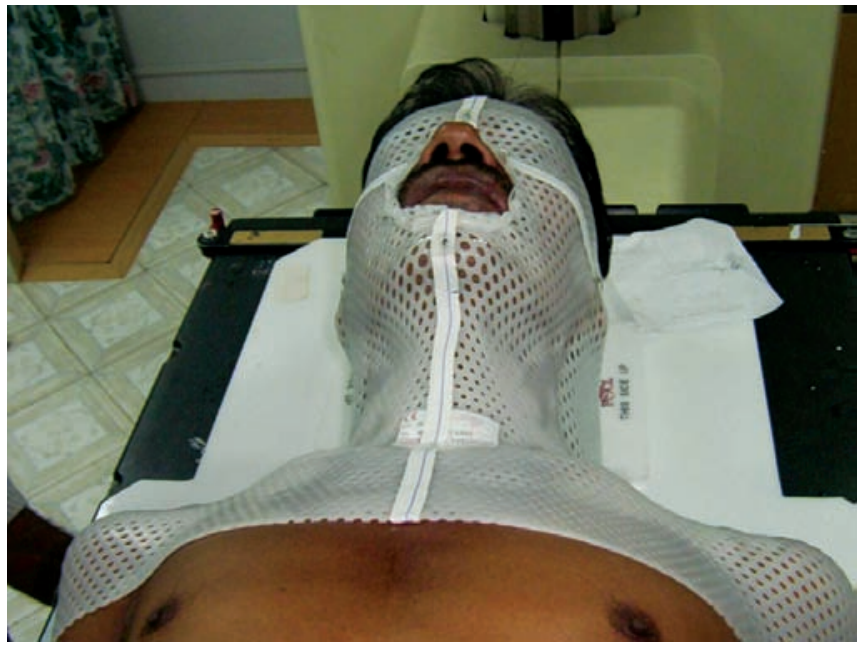

FIGURE 1: Patient in a thermoplastic mould

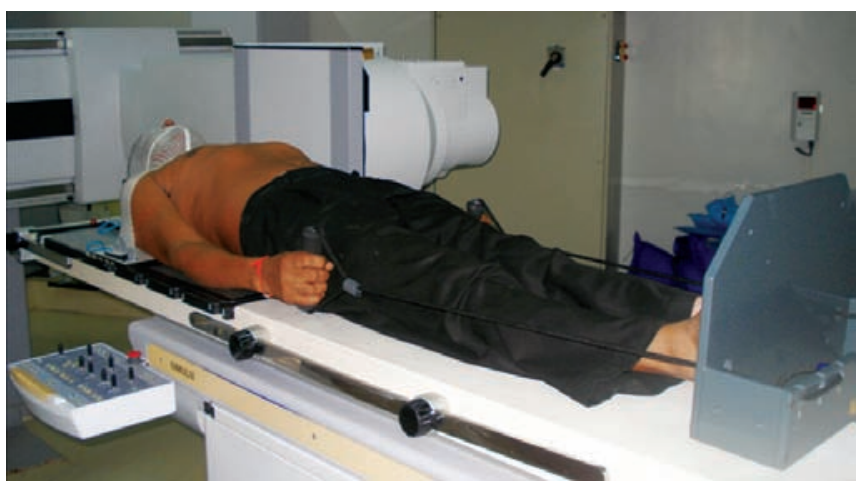

FIGURE 2: Patient immobilized on the simulator in the treatment position with mask and shoulder traction

\section{Simulation}

The simulator is a machine on which the patient can be positioned to 'simulate' the actual treatment. It enables the radiographical visualization of bony anatomy using X-rays and marking of the radiation beam (portal) on the mask (Figs 3 and 4).

Commonly 2 or 3 fields are used that cover the postoperative bed (primary and dissected neck) along with any areas of neck considered to be at high-risk of micro metastasis. The beam position depends on the site of the tumor and potential risk of spread to the lymph nodes in the neck.

\section{Dose Prescription}

The planned total dose is usually $60 \mathrm{~Gy}$ for margin negative disease. In patients with microscopically involved margins, the tumor bed receives a boost to $66 \mathrm{~Gy}$. Higher dose in such patient has shown to improve outcome. ${ }^{18}$ In patients 


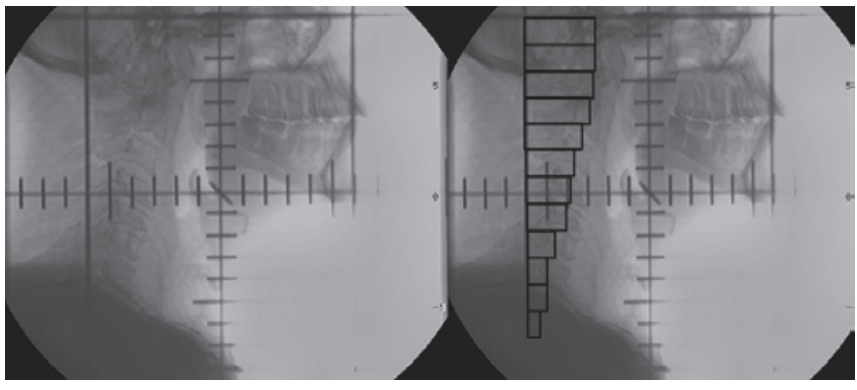

FIGURE 3: Simulator film showing lateral portals in relation to bony landmarks before and after spinal cord shielding

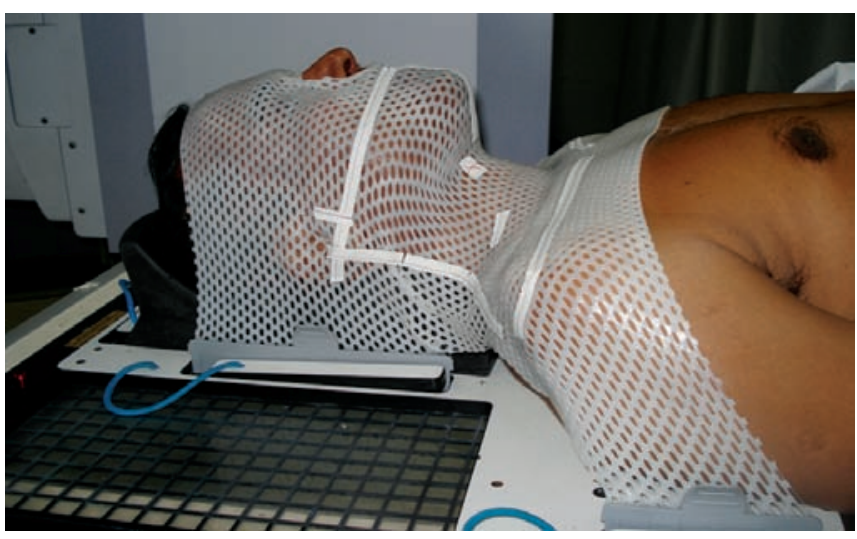

FIGURE 4: Patient in a thermoplastic mask showing the marked portals

with postlaryngectomy neck, the total dose is often reduced to limit normal tissue toxicity. ${ }^{19}$ Commonly, patients are treated with Telecobalt or 4 to $6 \mathrm{MV} \mathrm{X-rays} \mathrm{at} 2.0 \mathrm{~Gy}$ perfraction, one fraction per day, five days per week, over 6 weeks continuously. The treatment is planned and delivered in phases such that the radiation tolerance of critical structures like the spinal cord is respected (Fig. 3).

\section{Advanced Techniques of Radiotherapy}

Conventionally RT is delivered using 2 or 3 fields as described above. This involves treatment of a significant volume of normal tissues that come in the path of the radiation beams. The treatment planning is done using Orthogonal X-rays on the simulator using anatomical landmarks and is called 2dimensional radiotherapy technique.

Rapid in the last 2 decades in volumetric imaging modalities now allow the Radiation Oncologist to more accurately identify volumes and their relationship with other critical normal organs (Fig. 5). With powerful threedimensional treatment planning systems and advances in radiation treatment-delivery technology like the linear accelerator equipped with sophisticated computer-controlled

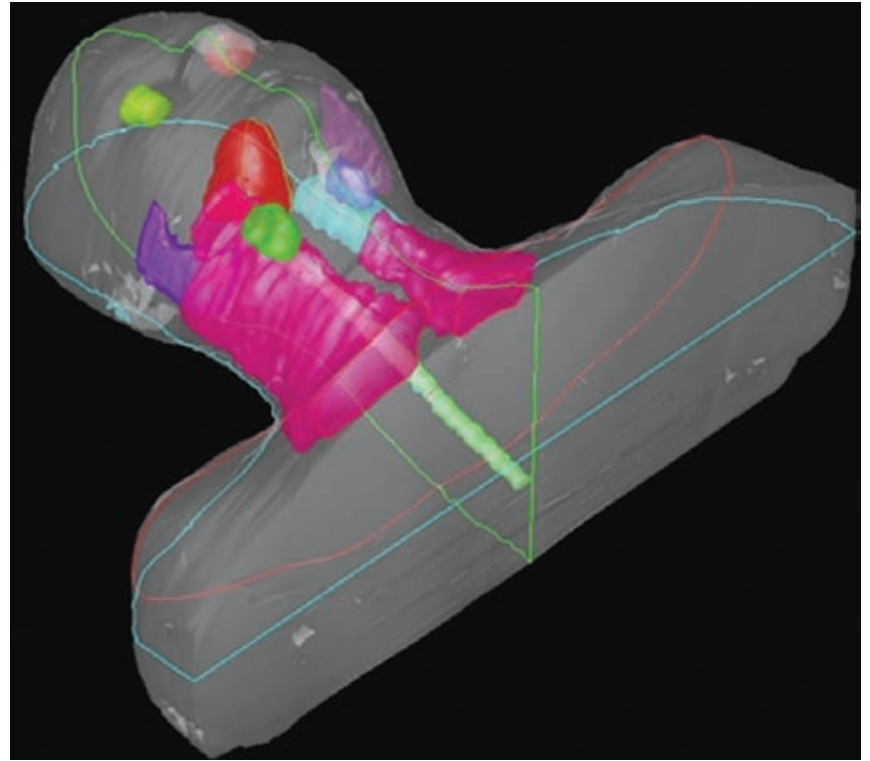

FIGURE 5: Contoured image showing the target volumes and the organs at risk (OAR)

multileaf collimator systems, three-dimensional conformal radiation therapy (3DCRT) is now commonly used to spare normal tissue and target the tumor better. In contrast to 2D treatment, 3DCRT treatment plans generally use an increased number of radiation beams (5-7) that are shaped to conform to the target volume (Fig. 6).

The next level of sophistication in the delivery of conformal radiotherapy is provided by intensity modulated radiotherapy (IMRT). IMRT is a technique that creates dose distributions that conform closely to the target volume and at the same time maximally sparing the organs at risk. This is done by optimally assigning nonuniform intensities or weights to tiny subdivisions of beams called beamlets. This computer-aided manipulation of the intensities of beamlets within each beam, permits increased control over the radiation fluence enabling highly conformal dose distributions (Fig. 7). These improved dose distributions potentially may lead to improved tumor control and reduced normal tissue toxicity. Although IMRT is considered as a current standard of care in the definitive chemoradiation setting, recent data suggests that the use of IMRT in the postoperative setting in head and neck cancers is associated with encouraging outcomes as well. ${ }^{20}$

\section{TOXICITY OF TREATMENT}

Due to the presence of critical structures related to taste, smell, mastication, swallowing and speech, the treatment of head and neck region with radiation is associated with 


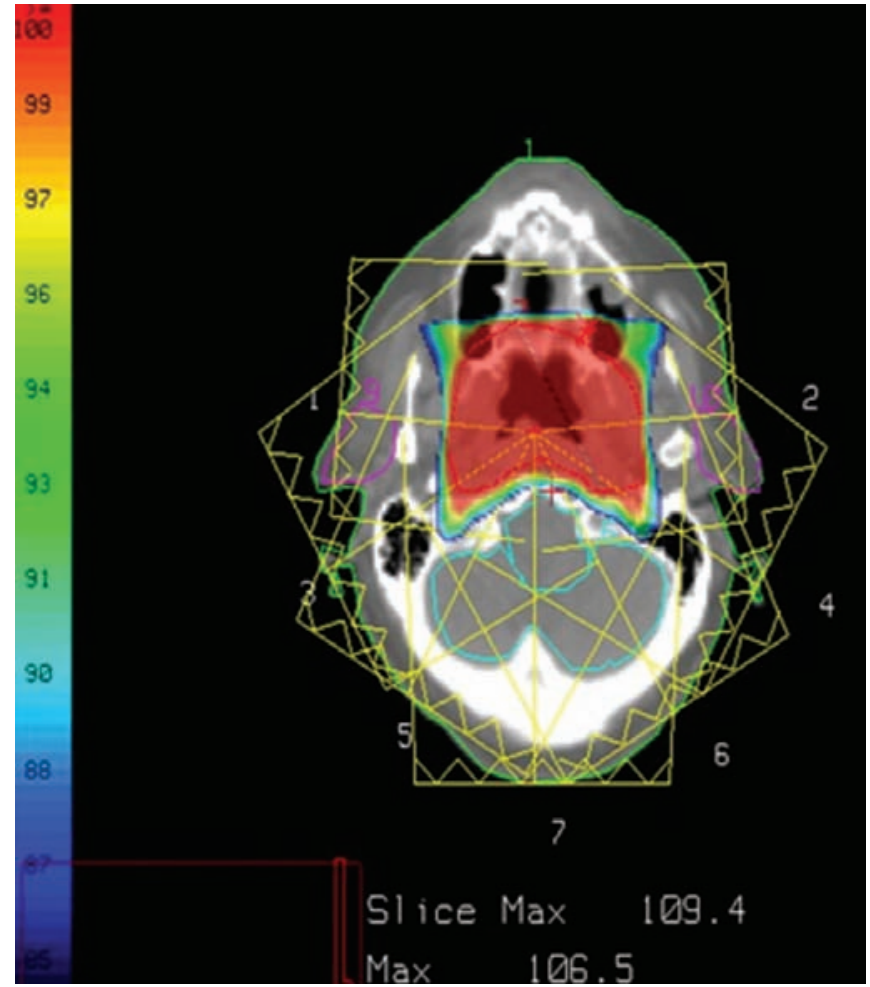

FIGURE 6: Axial CT image showing multiple beams used in conformal radiotherapy to focus the radiation to the target while sparing salivary glands (normal tissue)

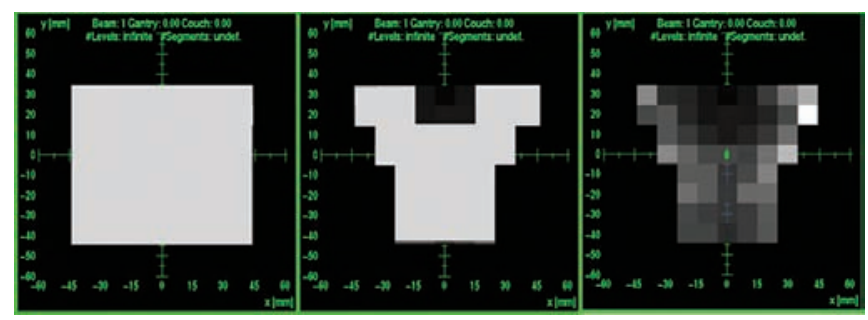

FIGURE 7: Fluence patterns of 2-dimensional (conventional) RT, 3D conformal radiation therapy (3DCRT) and intensity modulated radiation therapy (IMRT)

significant and often long standing sequelae. The use of modern technology and better understanding of the biological basis of these toxicities have somewhat helped in alleviating the side effects. During a course of head and neck radiation therapy, there are predictable side effects that are experienced by the majority of patients: mucositis, fatigue, loss of taste acuity, radiation dermatitis, and xerostomia. Usually acute effects appear after 1 to 3 weeks of treatment. Many patients experience general symptoms in the first week like fatigue and nausea which sometimes progresses as the treatment progresses. Nausea associated with treatment can also further complicate the nutritional status. Patients may also require prophylactic antiemetics. Mucositis manifests

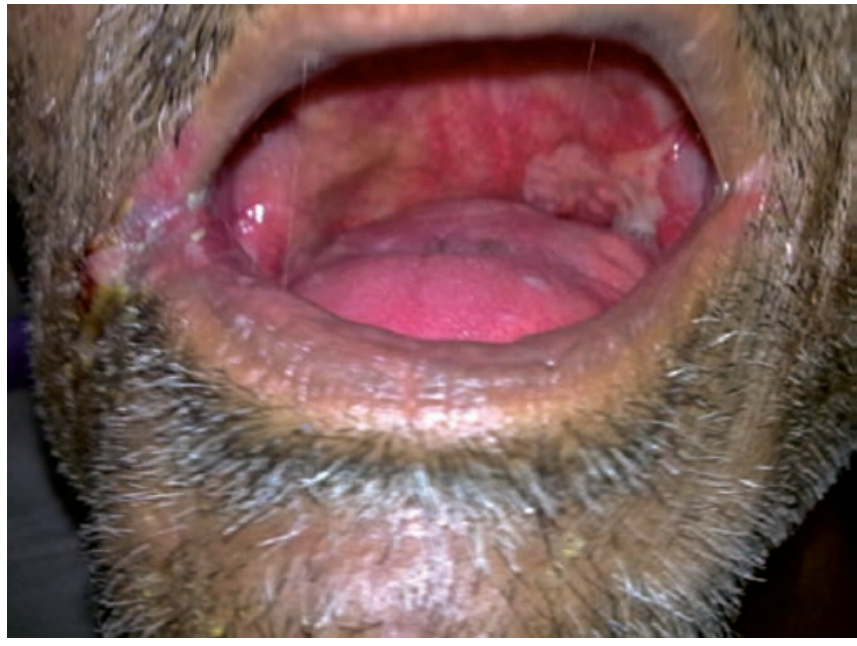

FIGURE 8: Patient undergoing radiation showing features of patchy mucositis in the 4th week of treatment

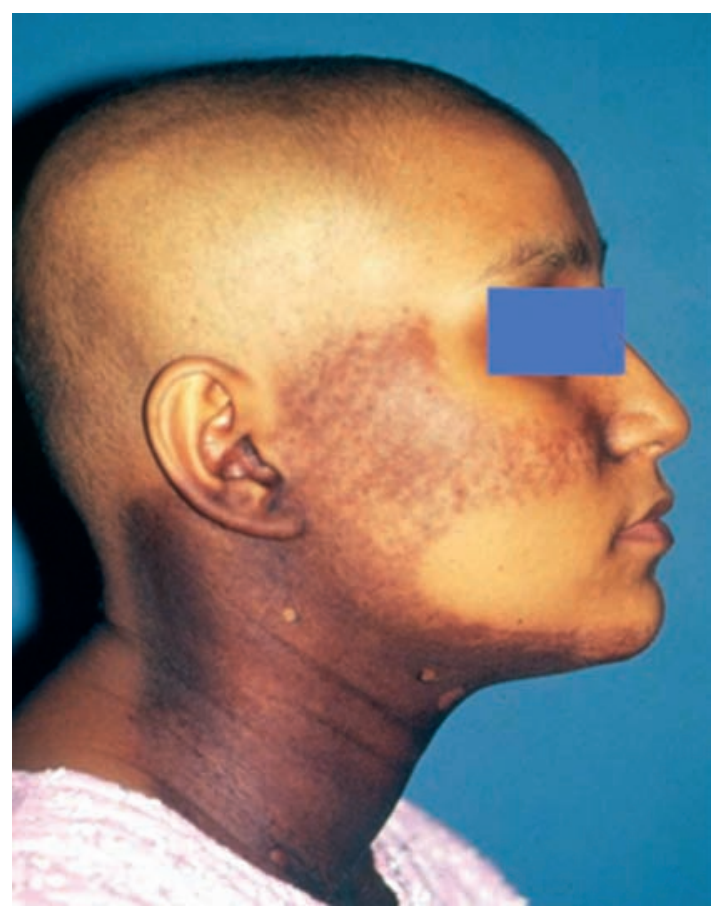

FIGURE 9: Patient undergoing RT showing skin changes of erythema/ pigmentation and dry desquamation in the 5 th week of treatment

as mucosal hyperemia within the treatment field, but can progress to patchy or confluent mucositis which represents denudation of the mucosal epithelium which generally regenerates after treatment (Fig. 8). Initially patients can be treated with NSAIDs, but they commonly require narcotic analgesics for adequate pain control. Signs or symptoms of infection (e.g thrush) should be looked for actively and treated promptly. Skin changes over a course of radiotherapy are well-documented and relatively easy to manage (Fig. 9). Xerostomia can be problematic due to 
interference with taste, speech, swallowing and mastication. These acute toxicities can become particularly pronounced in the setting of intensified radiation fractionation schedules and/or combined chemoradiotherapy. Ultimately most of these side effects, with the exception of xerostomia, are temporary and will resolve several weeks to months following completion of therapy.

Late toxicity occurs over 6 months to 2 years and is driven by underlying ischemia and fibrosis. Cell death in connective tissue attracts migrating fibroblasts which appear clinically as subcutaneous and submucosal fibrosis. This along with mild persistant xerostomia can promote dental caries, altered taste sensation, dysphagia and change of voice. Hypothyroidism, aspiration and osteoradionecrosis occur to varying degrees depending the final dose, fractionation schedule, concomitant treatment, severity of acute toxicity and medical comorbidity.

\section{ENHANCING EFFICACY: HOW TO IMPROVE TREATMENT OUTCOMES?}

\section{Role of Altered Fractionation Radiotherapy}

The standard fractionation schedule for most of the head and neck sites is 2 Gy, delivered once daily, 5 times a week. The biological behavior of head and neck cancer cells provides some scope for adjusting this schedule for clinical benefit. Two main approaches have been studied. The first, termed hyperfractionation, delivers two fractions a day separated by 6 hours or more, while delivering a reduced dose per fraction. This approach enables the delivery of a slightly higher total radiation dose to the tumor while not increasing the risk of late toxicity. The second, termed 'accelerated fractionation,' delivers more fractions of treatment per week by treating on weekends or delivering two or more fractions on some days of the week. This completes the delivery of radiation in a shorter and potentially reduces the impact of a fast repopulation of remaining tumor cells that occurs a few weeks into treatment.

The rationale behind altered fractionations has been derived from laboratory and clinical observations:

i. The reactions in the late reacting tissues (normal tissues) are much more dependent on size of dose per fraction than are acutely responding and tumor tissues. Hence, slowly responding tissues (responsible for late effects) can tolerate a higher total dose, provided the fraction size is shortened. ii. Tumor cells in the head and neck begin to replicate the clonogenic cells significantly above their pre-treatment levels after starting radiation. It is generally believed that the clonogenic cells begin to replicate significantly after 28 days of radiation and approximately $0.6 \mathrm{~Gy} /$ day is necessary to compensate for the tumor repopulation that transpires with each day of prolongation of standard course radiation. ${ }^{21}$

The therapeutic ratio can be increased by either or both approaches, increasing total dose through hyperfractionation or limiting the opportunity for tumor cell regeneration during treatment through accelerated fractionation.

Awwad and Colleagues ${ }^{22}$ randomized 70 patients with (T2/N1-N2) or (T3-4/any N) squamous cell carcinoma of the oral cavity, larynx and hypopharynx who underwent radical surgery. Patients were randomized to either (a) accelerated hyperfractionation: 46.2 Gy per 12 days, 1.4 Gy per fraction, three fractions per day with 6 hours interfraction interval, treating 6 days per week or (b) Conventional fractionation: 60 Gy per 6 weeks, 2 Gy per fraction, treating 5 days per week. The 3 -year LRC rate was significantly better in the accelerated hyperfractionation (88 $\pm 4 \%)$ than in the CF (57 $\pm 9 \%)$ group, $P=0.01$ (and this was confirmed by multivariate analysis), but the difference in survival $(60 \pm 10 \%$ vs $46 \pm 9 \%)$ was not significant $(P=0.29)$. They also suggested that to gain a full benefit from treatment acceleration, the surgeryradiotherapy gap and the overall treatment time should not exceed 6 and 10 weeks respectively.

Zouhair et $\mathrm{al}^{23}$ showed the feasibility and efficacy of accelerated PORT using a single daily fraction regimen from Mondays to Thursdays and a concomitant boost on Friday afternoon sessions in patients with locally advanced head and neck cancer. The total dose was66 Gy @ 2 Gy/\# over 5 weeks and 3 days. They demonstrated excellent local control and acceptable acute and late RT-related morbidity.

Another aspect of concern is the clinical impact of delay in start of adjuvant radiation. Huang et al. have shown that delay in initiation of radiation for more than 6 weeks after surgery is associated with significant increase in local recurrence rate (LRR) $(\mathrm{OR}=2.89 ; 95 \% \mathrm{CI}, 1.60$ to 5.21). There was little evidence about the impact of delay in radiation on the risk of metastases or the probability of longterm survival. ${ }^{24}$ Delays in starting radiation should be as small as reasonably achievable. 


\section{Addition of Chemotherapy}

Altering the fractionation does not have an impact on the probability of distant metastasis and hence overall survival. In the Swiss series, ${ }^{23}$ distant metastasis probability at 4 years was $38 \%$ (95\% CI 20-56). Postoperative chemotherapy has the potential to eradicate microscopic distant disease and enhance the effects of radiation as a sensitizer. To support the former hypothesis, Intergroup Study 0034 studied postoperative chemotherapy in a trial that randomized patients after surgery to three 21-day cycles of sequential cisplatin $\left(100 \mathrm{mg} / \mathrm{m}^{2}\right)$ and infusion 5-FU $(1,000$ $\mathrm{mg} / \mathrm{m}^{2} /$ day for 5 days) followed by PORT $v$ PORT alone..$^{25}$ There was no significant improvement in LRC or overall survival associated with the use of chemotherapy, but the incidence of distant metastases was reduced from 30 to $20 \%(\mathrm{p}=0.02)$.

The National Cancer Institute conducted a three-arm trial that evaluated the addition of one cycle of preoperative cisplatin and bleomycin with or without six cycles of sequential cisplatin $\left(80 \mathrm{mg} / \mathrm{m}^{2}\right)$ maintenance chemotherapy after surgery and postoperative irradiation. ${ }^{26}$ The control arm consisted of surgery and postoperative irradiation alone. This trial enrolled 443 patients and demonstrated no benefit with respect to LRC or survival from the addition of chemotherapy. Nearly half of the patients who were randomized to receive maintenance chemotherapy never received it. Despite this flaw in study execution, the incidence of distant metastases as site of first relapse was $9 \%$ in the patients assigned to maintenance chemotherapy as opposed to $19 \%$ in those who were not $(\mathrm{p}=0.02)$.

The role of concurrent chemotherapy along with postoperative radiation has been evaluated in 2 recent landmark prospective randomized trials by the Radiation Therapy Oncology Group (RTOG) and European Organization for Research and Treatment of Cancer (EORTC). In the RTOG study, ${ }^{27} 459$ patients with operable cancers of the oral cavity, oropharynx, larynx or hypopharynx were included. Eligibility criteria in the RTOG trial were patients with two or more positive nodes, extra capsular extension (ECE) and microscopically positive margins. Patients were randomized to postoperative radiation or postoperative chemoradiation (Cisplatin for 3 cycles on day $1,22,43)$. This trial demonstrated an improvement in LRC and disease free survival (DFS) for patients who received concurrent chemoradiotherapy. No significant benefit in absolute survival was confirmed. Expectedly, chemoradiation increased grade $3 / 4$ toxicities from 34 to $77 \%$.
The EORTC reported a similar trial that included 334 patients with stage III/IV cancers of the oral cavity, oropharynx, larynx or hypopharynx randomized to postoperative radiation $v s$ postoperative chemoradiation. ${ }^{28}$ This trial demonstrated a significant improvement in progression-free survival and overall survival with the addition of chemotherapy. Again, chemoradiotherapy was associated with increased toxicity (21 to $41 \%$ ).

A currently ongoing study at the Tata Memorial Centre is trying to address the optimal adjuvant treatment in oral cancer patients. Oral Cancer Adjuvant Therapy (OCAT) trial is recruiting approximately 900 patients into 3 arms in patients with high-risk features to either 'standard' RT (5 fractions a week), accelerated RT (6 fractions a week) or chemoradiotherapy (weekly cisplatin during RT). An interim analysis is due shortly and the results will help in better optimizing the adjuvant treatment in oral cancers (www.clinicaltrials.gov: NCT00193843).

Although the studies above identify that the addition of cisplatin chemotherapy to postoperative radiation can improve tumor control outcome for specific categories of high-risk patients, it is clear that this modest benefit comes at the expense of additional toxicity. Careful clinical judgment regarding the selection of patients most likely to tolerate and thereby benefit from this approach is warranted. In the definitive treatment setting, there is mounting evidence that patients $>70$ years of age derive little benefit from the addition of systemic chemotherapy to radiation in head and neck cancer. ${ }^{29}$ This is likely to be true in the postoperative head and neck cancer treatment setting as well. The inadvertent introduction of treatment breaks during the adjuvant radiation course can easily compromise the potential benefits of the combined modality therapy in this setting.

\section{Addition of Targeted Therapies}

Targeted therapy in locally advanced head and neck cancer consists of monoclonal antibodies. The main objective is to stimulate the patient's immune system to attack the malignant cells and prevention of tumor growth by blocking specific cell receptors. These are newer classes of promising drugs that target molecular structures or pathways unique to the tumor.

Monoclonal antibody against the epidermal growth factor receptors (EGFR) has been studied in locally advanced head and neck cancers because epidermal growth factor receptors are found in plenty in these tumors leading to cellular proliferation and tumor growth. Cetuximab is the prototype 
chimeric monoclonal antibody against EGFR. A large multicentric phase III trial by Bonner et al compared definitive radiation alone $v s$ radiation plus cetuximab in advanced head and neck cancer patients. Addition of cetuximab showed improvement in LRC (34 to 47\%) and overall survival (45 to 55\%). With the exception of acneiform rash and infusion reactions with cetuximab, toxicity was similar. ${ }^{30}$ The trial by Bonner et al was criticized for the fact that it compared radiation alone with radiation plus cetuximab. Ongoing trials to examine the potential value of adding cetuximab to concurrent chemoradiation approaches in advanced head and neck cancer are in progress in both the definitive and high-risk postoperative settings.

Recently, another new humanized monoclonal antibody against the EGFR is available commercially. Nimotuzumab has high affinity and specificity for EGFR and exhibits antiproliferative, proapoptotic and antiangiogenic effect. Preliminary efficacy, safety and pharmacodynamic profile favours the use of nimotuzumab concurrently with radiation in inoperable locally advanced head and neck cancers. ${ }^{31,32}$ Ongoing and planned trials will demonstrate whether nimotuzumab will also be efficacious in the adjuvant setting.

\section{CONCLUSION}

In summary, head and neck cancer patients with unfavorable factors have an increased risk of locoregional failure after surgery, and postoperative RT improves outcome. Intensification of treatment with addition of platinum based chemotherapy is now considered standard in a subset of patients at a particularly high-risk of relapse. Overall treatment time should be minimised by avoiding delay between the date of surgery and end of radiotherapy. Accelerated radiotherapy is also a promising method of treatment intensification albeit at a risk of higher toxicity. Careful planning and delivery of radiotherapy and use of modern delivery methods go a long way in improving the therapeutic ratio.

\section{REFERENCES}

1. Sanghvi LD, Rao DN, Joshi S. Epidemiology of head and neck cancers. Semin Surg Oncol1989;5(5):305-09.

2. Mendenhall WM, Morris CG, Amdur RJ, Hinerman RW, Mancuso AA. Parameters that predict local control after definitive radiotherapy for squamous cell carcinoma of the head and neck. Head Neck Jul 2003;25(7):535-42.

3. Huang DT, Johnson CR, Schmidt-Ullrich R, Grimes M. Postoperative radiotherapy in head and neck carcinoma with extracapsular lymph node extension and/or positive resection margins: A comparative study. Int J Radiat Oncol Biol Phys1992;23(4):737-42.

4. Lundahl RE, Foote RL, Bonner JA, Suman VJ, Lewis JE, Kasperbauer JL, et al. Combined neck dissection and postoperative radiation therapy in the management of the highrisk neck: A matched-pair analysis. Int J Radiat Oncol Biol Phys Feb 19981;40(3):529-34.

5. Pignon JP, Bourhis J, Domenge C, Designe L. Chemotherapy added to locoregional treatment for head and neck squamous-cell carcinoma: Three meta-analyses of updated individual data. MACH-NC Collaborative Group. Meta-Analysis of Chemotherapy on Head and Neck Cancer. Lancet Mar 2000;18;355(9208):949-55.

6. Gleich LL, Ryzenman J, Gluckman JL, Wilson KM, Barrett WL, Redmond KP. Recurrent advanced (T3 or T4) head and neck squamous cell carcinoma: Is salvage possible? Arch Otolaryngol Head and Neck Surg Jan 2004;130(1):35-38.

7. Maccomb WS, Fletcher GH. Planned combination of surgery and radiation in treatment of advanced primary head and neck cancers. Am J Roentgenol Radium Ther Nucl Med Mar 1957;77(3):397-414.

8. Kramer S, Gelber RD, Snow JB, Marcial VA, Lowry LD, et al. Combined radiation therapy and surgery in the management of advanced head and neck cancer: Final report of study 73-03 of the Radiation Therapy Oncology Group. Head and Neck Surg Sep-Oct 1987;10(1):19-30.

9. Peters LJ, Goepfert H, Ang KK, Byers RM, Maor MH, et al. Evaluation of the dose for postoperative radiation therapy of head and neck cancer: First report of a prospective randomized trial. Int J Radiat Oncol Biol Phys Apr 1993;30;26(1):3-11.

10. Ang KK, Trotti A, Brown BW, Garden AS, Foote RL, et al. Randomized trial addressing risk features and time factors of surgery plus radiotherapy in advanced head and neck cancer. Int J Radiat Oncol Biol Phys Nov 2001;1;51(3):571-78.

11. Cooper JS, Pajak TF, Forastiere A, Jacobs J, Fu KK, et al. Precisely defining high-risk operable head and neck tumors based on RTOG \#85-03 and \#88-24: targets for postoperative radiochemotherapy? Head and Neck Oct 1998;20(7):588-94.

12. An SY, Jung EJ, Lee M, Kwon TK, Sung MW, Jeon YK, et al. Factors related to regional recurrence in early stage squamous cell carcinoma of the oral tongue. Clin Exp Otorhinolaryngol Sep 2008;1(3):166-70.

13. Fakih AR, Rao RS, Borges AM, Patel AR. Elective versus therapeutic neck dissection in early carcinoma of the oral tongue. Am J Surg Oct 1989;158(4):309-13.

14. O'Brien CJ, Lauer CS, Fredricks S, Clifford AR, McNeil EB, Bagia JS, et al. Tumor thickness influences prognosis of T1 and T2 oral cavity cancer-but what thickness? Head and Neck Nov 2003;25(11):937-45.

15. Fukano H, Matsuura H, Hasegawa Y, Nakamura S. Depth of invasion as a predictive factor for cervical lymph node metastasis in tongue carcinoma. Head and Neck May 1997;19(3):205-10.

16. Mishra RC, Singh DN, Mishra TK. Postoperative radiotherapy in carcinoma of buccal mucosa, a prospective randomized trial. Eur J Surg Oncol Oct 1996;22(5):502-04.

17. Frank JL, Garb JL, Kay S, McClish DK, Bethke KP, et al. Postoperative radiotherapy improves survival in squamous cell carcinoma of the hypopharynx. Am J Surg Nov 1994;168(5):476-80. 
18. Pfreundner L, Willner J, Marx A, Hoppe F, Beckmann G, Flentje M. The influence of the radicality of resection and dose of postoperative radiation therapy on local control and survival in carcinomas of the upper aerodigestive tract. Int J Radiat Oncol Biol Phys2000 Jul 15;47(5):1287-97.

19. Lee NK, Goepfert H, Wendt CD. Supraglottic laryngectomy for intermediate-stage cancer: UTMD. Anderson Cancer Center experience with combined therapy. Laryngoscope Aug 1990;100(8):831-36.

20. Studer G, Furrer K, Davis BJ, Stoeckli SS, Zwahlen RA, Luetolf UM, et al. Postoperative IMRT in head and neck cancer. Radiat Oncol 2006;1:40.

21. Withers HR, Taylor JM, Maciejewski B. The hazard of accelerated tumor clonogen repopulation during radiotherapy. Acta Oncol 1988;27(2):131-46.

22. Awwad HK, Lotayef M, Shouman T, Begg AC, Wilson G, Bentzen SM, et al. Accelerated hyperfractionation (AHF) compared to conventional fractionation $(\mathrm{CF})$ in the postoperative radiotherapy of locally advanced head and neck cancer: Influence of proliferation. Br J Cancer Feb 2002;12;86(4):517-23.

23. Zouhair A, Azria D, Pasche P, Stupp R, Chevalier J, et al. Accelerated postoperative radiotherapy with weekly concomitant boost in patients with locally advanced head and neck cancer. Radiother Oncol Feb 2004;70(2):183-88.

24. Huang J, Barbera L, Brouwers M, Browman G, Mackillop WJ. Does delay in starting treatment affect the outcomes of radiotherapy? A systematic review. J Clin Oncol Feb 2003;1;21(3):555-63.
25. Laramore GE, Scott CB, al-Sarraf M, Haselow RE, Ervin TJ, et al. Adjuvant chemotherapy for resectable squamous cell carcinomas of the head and neck: Report on Intergroup Study 0034. Int J Radiat Oncol Biol Phys 1992;23(4):705-13.

26. Adjuvant chemotherapy for advanced head and neck squamous carcinoma. Final report of the Head and Neck Contracts Program. Cancer Aug 1987;1;60(3):301-11.

27. Cooper JS, Pajak TF, Forastiere AA, Jacobs J, Campbell BH, et al. Postoperative concurrent radiotherapy and chemotherapy for high-risk squamous-cell carcinoma of the head and neck. $\mathrm{N}$ Engl J Med May 2004;6;350(19):1937-44.

28. Bernier J, Domenge C, Ozsahin M, Matuszewska K, Lefebvre $\mathrm{JL}$, et al. Postoperative irradiation with or without concomitant chemotherapy for locally advanced head and neck cancer. $\mathrm{N}$ Engl J Med May 2004;6;350(19):1945-52.

29. Pignon JP, le Maitre A, Bourhis J. Meta-Analyses of Chemotherapy in Head and Neck Cancer (MACH-NC): An update. Int J Radiat Oncol Biol Phys 2007;69(2 Suppl):S112-4.

30. Bonner JA, Harari PM, Giralt J, Azarnia N, Shin DM, et al. Radiotherapy plus cetuximab for squamous-cell carcinoma of the head and neck. N Engl J Med Feb 2006;9;354(6):567-78.

31. Combet T, Osorio M, Cruz T, et al. Efficacy evaluation of the humanized anti-EGFR MAb h-R3 (nimotuzumab) in combination with radiotherapy in the treatment of patients with unresectable squamous cell carcinomas of the head and neck. Eur J Cancer 2006;4:62.

32. Reddy BK, Vidyasagar M, Shenoy K, et al. BioMAb EGFRTM (Nimotuzumab/h-R3) in combination with standard of care in squamous cell carcinoma of head and neck. Int J Radiat Oncol Biol Phys 2007;69(1):S450. 